\title{
Topical anaesthesia of the vocal cords by nebulized lignocaine inhalation to facilitate fibreoptic nasotracheal intubation in a head-size parotid tumour patient
}

\author{
Koirala S, ${ }^{1}$ Tripathi $M{ }^{2}$ Subedi A, ${ }^{1}$ Bhattarai $B^{1}$ \\ 'Department of Anaesthesiology and Critical Care, B.P. Koirala Institute of Health Sciences, Dharan, Nepal, ${ }^{2}$ Department of \\ Anesthesiology, SGPGIMS, Lucknow, India
}

\section{ABSTRACT}

This case report describes a successful fibreoptic naso-tracheal intubation in a patient with difficult airway due to a nearly head-size right parotid swelling. Nasal fibreoptic intubation was performed by maintaining spontaneous breathing under propofol infusion at a dose of $25 \mathrm{mcg} / \mathrm{kg} / \mathrm{hour}$ and topical anaesthesia of vocal cords by using oxygen $(6 \mathrm{l} / \mathrm{min})$ flow to nebulize $4 \%$ lignocaine from the side port of the fibreoptic bronchoscope. The topical anaesthesia was achieved by keeping the scope in close proximity of the vocal cords and in the centre of view of the scope. This case emphasizes the possibility of fibreoptic intubation in a sedated yet spontaneously breathing patient by allowing inhalation of nebulized lignocaine during fibreoptic intubation.

Keywords: fibreoptic intubation, naso-tracheal intubation, nebulized

lignocaine, spontaneous breathing, topical anaesthesia

\section{INTRODUCTION}

Studies have emphasized the need of retaining spontaneous breathing during flexible fibreoptic intubation (FOI) in patients with compromised airways. ${ }^{1-3}$ Instead of intubating an awake patient after nerve blocks, a conscious sedative technique for nasotracheal intubation is now propagated. ${ }^{4}$ Topical lignocaine has also been described in supressing coughing during bronchoscopy ${ }^{5}$ and FOI. ${ }^{6}$ These techniques have been successfully reported in patients with normal airway but reports of intubation in a real time difficult situation are very few in the literature. We wish to share our experience of successfully managing nasal $\mathrm{FOI}$ in spontaneously breathing patient having a handicap for laryngeal nerve blocks due to a huge parotid mass distorting the anatomical landmarks in the neck.

\section{CASE REPORT}

A 62-year-old lady weighing $48 \mathrm{~kg}$ presented with a nearly head size swelling on the right side of neck and cheeks. The patient was apparently asymptomatic 15 years back when she developed a swelling over the right cheek. It was painless and gradually increased in size. There was no difficulty in swallowing, eye movement or breathing. There was a rapid increase in size during the last one month with appearance of mild pain, itching and difficulty in chewing from the right side of the mouth.

On examination, a large multinodular swelling $(20 \times 14$ $x 16 \mathrm{cms}$ ) was noted on the right cheek extending from the right ear to right supraclavicular region. Posteriorly, the swelling extended up to the occipital region and anteriorly up to the angle of the mouth. The patient was unable to move her face to the right side and also had difficulty in stretching her neck (Figure 1).

Computerized tomography of the head and neck revealed a huge parotid mass infiltrating up to parapharyngeal space compressing the right internal jugular and the carotid artery, extending below up to the supraclavicular

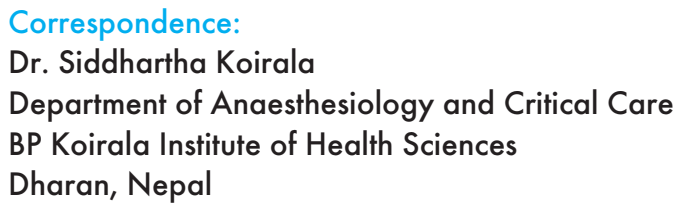


fossa but sparing the thoracic outlet. It had dislocated the right temporo-mandibular joint and distorted the oro-pharyngeal cavity (Figure 2). Fine needle aspiration confirmed pleomorphic adenoma of the parotid with malignant transformation.

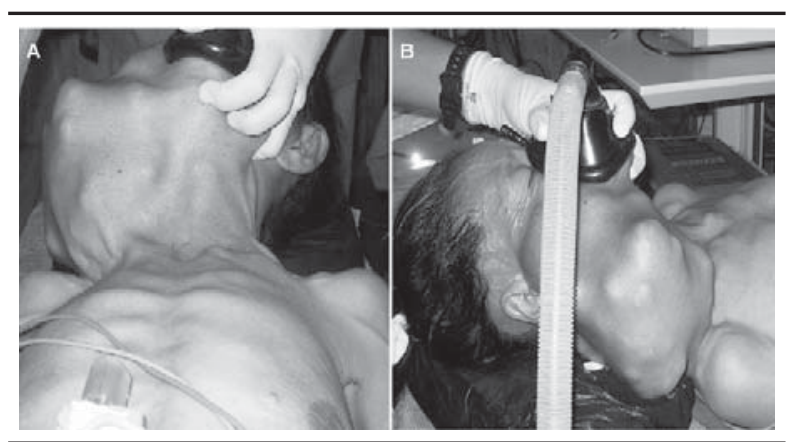

Figure 1. A-front and B-side view of parotid tumour occupying whole of the right neck from the angle of mouth till supraclavicular space.

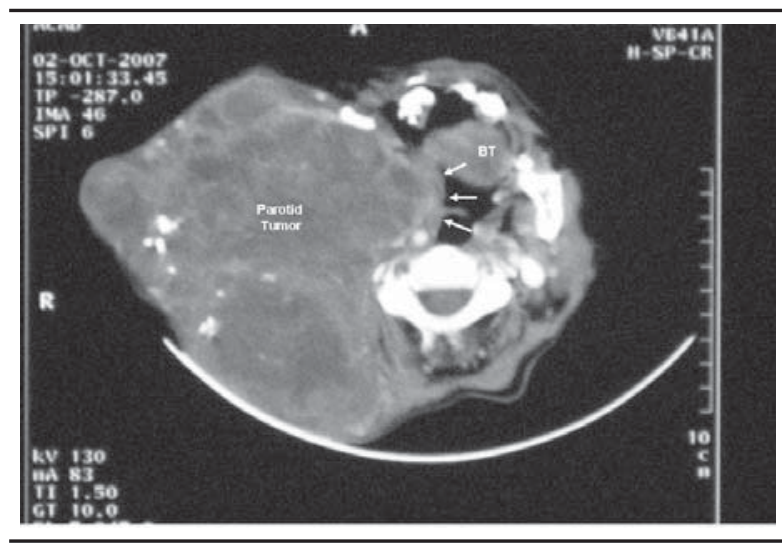

Figure 2. CT scan at the level of mouth showing tumour bulging inside oropharynx marked by the arrows was causing obstructing direct laryngoscope and orotracheal intubation.

Airway assessment showed restricted mouth opening $(2 \mathrm{~cm})$, Mallampati grade IV, and shifting of the trachea leftward from the midline. Thus, we anticipated difficult intubation and possibly difficult bag and mask ventilation after muscle paralysis. The patient was planned for parotidectomy and was given lorazepam tablet $(1 \mathrm{mg}$ ) the night before and two hours before surgery.

The airway management strategy desired was the maintenance of spontaneous breathing not losing airway control till securing the tracheal intubation. A naso-tracheal intubation with the help of fibreoptic bronchoscope was planned. Devices like a laryngeal mask airway and a tracheotomy procedure were kept at standby in case airway obstruction developed.

The patient was monitored as per standard protocol. In the left nostril, $4 \%$ lignocaine jelly was instilled to lubricate and anesthetize the nasal passage. Glycopyrrolate $(0.01$ $\mathrm{mg} / \mathrm{kg}$ ) was given intravenously to reduce secretions. Injection pethidine $(40 \mathrm{mg}$ ) was given intravenously. The patient was preoxygenated with $100 \%$ oxygen for three minutes and then propofol infusion at a dose of $25 \mu \mathrm{g} / \mathrm{kg} / \mathrm{hour}$ was started. After no response to verbal command, the fibreoptic bronchoscope was inserted through the patent left nare. The patient maintained spontaneous breathing during propofol infusion. Oxygen insufflation $(6 \mathrm{l} / \mathrm{min})$ through a nebulizer was started to administer a fine mist of $4 \%$ lignocaine through the side-port of the fibreoptic bronchoscope. Two millilitres of $4 \%$ lignocaine was used in the nebulizer. The tip of the bronchoscope with a diameter of $4.5 \mathrm{~mm}$ was placed just beyond the epiglottis while keeping the vocal cords in the centre view of the scope for one minute. This allowed inhalation of oxygen and the lignocaine mist to anesthetize the vocal cords of the patient (Figure 3).

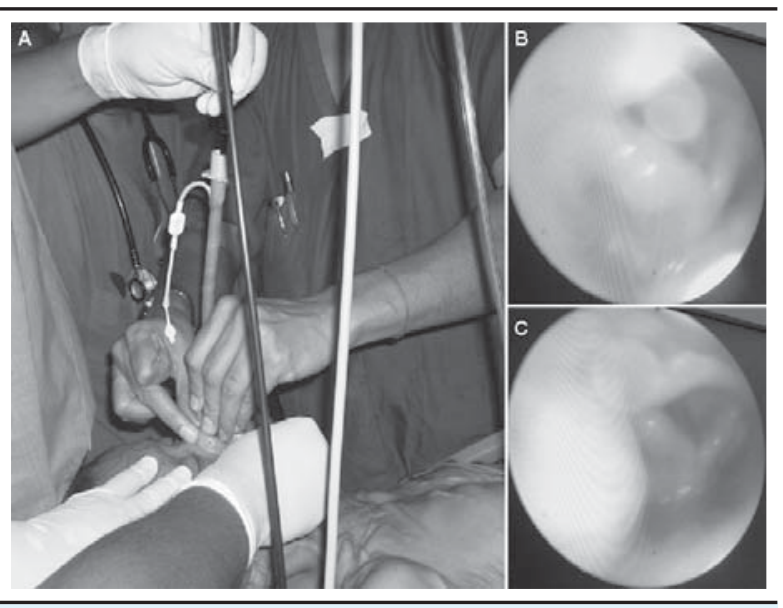

Figure 3. A-nasotracheal intubation being performed using flexometanlic tube over the fibreoptic bronchoscope; B-lignocaine mist developing during expiration, C-mist is cleared on inspiration.

The patient maintained breathing, airway and oxygen saturation during topical anaesthesia of the vocal cords. She neither had coughing nor desaturation below 98 $\%$. As the vocal cords gradually stopped moving with inspiration, the scope was negotiated inside the trachea till the carina was visualized. At this point, a flexometalic tube $(7.0 \mathrm{~mm})$ was railroaded over the bronchoscope (Figure $3 \mathrm{~A}$ ). It stimulated mild coughing and tachycardia (128 bpm). The time to complete the intubation was 268 seconds. After tracheal intubation, we maintained anesthesia with vecuronium $(6 \mathrm{mg})$ and isoflurane $(1.5$ $\%)$ during surgery, which was uneventful. The patient was discharged after two weeks.

\section{DISCUSSION}

Nasotracheal fibreoptic intubation is an awake patient is an established method of securing the airway in a 
Koirala et al. Lignocaine inhalation for fibreoptic intubation

difficult intubation setting. Local and regional anesthesia techniques facilitate intubation in awake patients. However, due to the huge swelling in the neck distorting the land marks, it was difficult to give a superior laryngeal nerve or intratracheal block to our patient. So we evolved a sedative technique with the help of propofol infusion with retained spontaneous breathing. Inhalation of the mist of nebulized lignocaine in oxygen through the fiberscope allowed topical anesthesia of vocal cords.

Smith et $\mathrm{al}^{2}$ suggested $\mathrm{FOl}$ in anesthetized spontaneously breathing patients where the time to perform FOI is not restricted by apnea, a fact which may be particularly advantageous in patients with a difficult airway, as in our patient. Ideally, the FOI technique under sedation should provide patient comfort, ensure good airway reflex suppression under sufficient spontaneous breathing, hemodynamic stability, and adequate intubating conditions. ${ }^{4}$ Halothane $^{2}$ and sevoflurane ${ }^{3}$ have been described to facilitate FOI in spontaneous breathing patients. But a high, around $30 \%$, incidence of hypoxemia $\left(\mathrm{SpO}_{2}<90 \%\right)$ was reported with halothane in $50 \%$ oxygen given via Mathias oral airway during FOI. ${ }^{2}$ Desaturation episodes were seen during difficulty in maintaining a patent airway and depth of anesthesia.
Since we used propofol by intravenous infusion, the depth of anasthesia and breathing were well maintained. Moreover, in our technique, we utilized the side channel of FOl to deliver a continuous oxygen flow along with lignocaine aerosol in close proximity to the vocal cords, which helped us to oxygenate our spontaneously breathing patient and at the same time topically anesthetized vocal cords. Only mild coughing was seen during insertion of the endotracheal tube.

The safety of oxygen to nebulize lignocaine has been very well studied ${ }^{7}$ and described to suppress the coughing reflex during bronchoscopy. ${ }^{8}$ We too found that the nebulization of lignocaine by oxygen flow of $6 \mathrm{l} / \mathrm{min}$ from the anesthesia machine was effective in maintaining oxygenation in a sedated and breathing patient.

Although we have found this method of fibreoptic intubation which allowed spontaneous breathing under sedation without a need to put nerve blocks, losing airway control or oxygenation for a prolonged intubation time (more than four minutes) very encouraging, further studies are warranted to assess this technique for routine fibreoptic intubation.

\section{REFERENCES}

1. Benumof JL. Management of the difficult adult airway. With special emphasis on awake tracheal intubation. Anesthesiology. 1991;75:1087-110.

2. Smith M, Calder I, Crockard A, Isert P, Nicol ME. Oxygen saturation and cardiovascular changes during fibreoptic intubation under general anaesthesia. Anaesthesia. 1992;47:158-61.

3. Erb T, Hampl KF, SchÜrch M, Kern CG, Marsch SC. Teaching the use of fibreoptic intubation in anesthetized, spontaneously breathing patients. Anesth Analg. 1999;89:1292-5.

4. Machata AM, Gonano C, Holzer A, et al. Awake nasotracheal fibreoptic intubation: patient comfort, intubating conditions, and hemodynamic stability during conscious sedation with remifentanil. Anesth Analg. 2003;97:904-8.
5. Jakobsen CJ, Ahlburg P, Holdgård HO, Olsen KH, Thomsen A. Comparison of intravenous and topical lidocaine as a suppressant of coughing after bronchoscopy during general anaesthesia. Acta Anaesthesiol Scand. 1991;35:238-41.

6. Wieczorek PM, Schricker T, Vinet B, Backman SB. Airway topicalisation in morbidly obese patients using atomised lidocaine: $2 \%$ compared with $4 \%$. Anaesthesia. 2007;62:984-8.

7. Tsui BCh, Malherbe S. Nebulization of lidocaine with varied oxygen flow rates. Anesth Analg. 2003;97:302.

8. Gjonaj ST, Lowenthal DB, Dozor AJ. Nebulized lidocaine administered to infants and children undergoing flexible bronchoscopy. Chest. 1997;112:1165-9. 